\title{
Atom trap and waveguide using a two-color evanescent light field around a subwavelength-diameter optical fiber
}

\author{
Fam Le Kien, ${ }^{1, *}$ V. I. Balykin, ${ }^{1,2}$ and K. Hakuta ${ }^{1}$ \\ ${ }^{1}$ Department of Applied Physics and Chemistry, University of Electro-Communications, Chofu, Tokyo 182-8585, Japan \\ ${ }^{2}$ Institute of Spectroscopy, Troitsk, Moscow Region, 142092, Russia
}

(Dated: November 4, 2018)

\begin{abstract}
We suggest using a two-color evanescent light field around a subwavelength-diameter fiber to trap and guide atoms. The optical fiber carries a red-detuned light and a blue-detuned light, with both modes far from resonance. When both input light fields are circularly polarized, a set of trapping minima of the total potential in the transverse plane is formed as a ring around the fiber. This design allows confinement of atoms to a cylindrical shell around the fiber. When one or both of the input light fields are linearly polarized, the total potential has two local minimum points in the transverse plane. This design allows confinement of atoms to two straight lines parallel to the fiber axis. Due to the thin thickness of the fiber, we can use far-off-resonance fields with substantially differing evanescent decay lengths to produce a net potential with a large depth, a large coherence time, and a large trap lifetime. For example, a $0.2-\mu \mathrm{m}$-radius silica fiber carrying $30 \mathrm{~mW}$ of 1.06 $\mu \mathrm{m}$-wavelength light and $29 \mathrm{~mW}$ of $700-\mathrm{nm}$-wavelength light, both fields are circularly polarized at the input, gives for cesium atoms a trap depth of $2.9 \mathrm{mK}$, a coherence time of $32 \mathrm{~ms}$, and a recoil-heating-limited trap lifetime of $541 \mathrm{~s}$.
\end{abstract}

PACS numbers: 32.80.Pj,32.80.Lg,03.75.Be,03.65.Ge

\section{INTRODUCTION}

One of the key problems of matter-wave physics is trapping and guiding neutral atoms [1]. Atom traps and atom waveguides can be used as tools for atom optics, atom interferometry, and atom lithography [2]. Atoms can be trapped and manipulated by the gradient forces of light waves [3]. In particular, the evanescent light waves have been used extensively to trap and guide atoms since they have high spatial gradients and use rigid dielectric structures like prisms and fibers to define the potential shape [4, 5, 6, 7]. An example is a hollow optical fiber with light propagating in the glass and tuned far to blue of atomic resonance 7]. Inside the fiber, the evanescent wave decays exponentially away from the wall, producing a repulsive potential which guides atoms along the center axis. Alternatively, a red-detuned light in the hollow center of the fiber can also be used to guide atoms $[6]$. In several experiments [5], cold atoms have been trapped and guided inside a hollow fiber.

Recently, a method for trapping and guiding neutral atoms outside a thin optical fiber has been proposed [8]. The scheme is based on the use of a subwavelengthdiameter silica fiber with a red-detuned light launched into it. The light wave decays away from the fiber wall and produces an attractive potential for neutral atoms. To sustain a stable trapping and guiding outside the fiber, the atoms have to be kept away from the fiber wall. This can be achieved by a centrifugal potential barrier 8].

Another way to produce a trap with a potential minimum outside a surface is to employ the idea of Ovchinnikov et al. [9], who proposed the use of two colors (i.e., red and blue detunings) and differing evanescent decay lengths to obtain both attractive and repulsive forces. The two-color method has been considered for planar prisms [9], dielectric microspheres [10], freestanding channel waveguides [11, and integrated optical waveguides 12]. In these systems, the net trapping potential is small compared to the optical potentials of the red- and blue-detuned lights. Such traps are sensitive to small field perturbations. As already pointed out by Barnett et al. 11], such sensitivity would be greatly reduced if we could increase the difference between the evanescent decay lengths of the red- and blue-detuned lights. In addition, utilizing large detunings would also be advantageous for coherent guiding 11, 12].

In this paper, we demonstrate that a subwavelengthdiameter optical fiber carrying a red-detuned light and a blue-detuned light can be used to trap and guide atoms outside the fiber. Due to the thin thickness of the fiber, we can use far-off-resonance lights with substantially differing evanescent decay lengths to produce a net potential with (1) a deep minimum, (2) a large coherence time, and (3) a large trap lifetime. We consider two schemes of input field polarization. In the scheme where both light fields are circularly polarized at the input, a set of trapping minima of the potential in the transverse plane is formed as a ring around the fiber, and the atoms can be confined to a cylindrical shell around the fiber. In the scheme where one or both of the input light fields are linearly polarized, the potential has two local minimum points in the transverse plane, and the atoms can be confined along two straight lines parallel to the fiber axis.

Before we proceed, we note that, due to recent developments in taper fiber technology, thin fibers can be produced with diameters down to $50 \mathrm{~nm}$ [13, 14]. Therefore, a two-color trap using a subwavelength-diameter fiber is a quite realistic design. In addition to the potential practical applications for trapping and guiding 
of atoms, a tapered fiber with an intense evanescent field can also be used as an atomic mirror [15]. Generation of light with a supercontinuum spectrum in thin tapered fibers has been demonstrated [14]. The evanescent waves from zero-mode metal-clad subwavelengthdiameter waveguides have been used for optical observations of single-molecule dynamics [16]. Rigorous calculations for the spatial distribution, the waveguide dispersion, and the polarization orientation of the light field around a thin fiber have been reported [15, 17, 18]. Thin fiber structures can be used as building blocks in the future atom and photonic micro and nano devices.

The paper is organized as follows. In Sec. [II we study the scheme where both input light fields are circularly polarized. In Sec. III we study the scheme where one or both of the input light fields are linearly polarized. Our conclusions are given in Sec. IV

\section{TWO-COLOR TRAP WITH CIRCULARLY POLARIZED INPUT LIGHTS}

Consider a thin single-mode optical fiber that has a cylindrical silica core of radius $a$ and refractive index $n_{1}$ and an infinite vacuum clad of refractive index $n_{2}=1$. Such a fiber can be prepared using taper fiber technology. The essence of the technology is to heat and pull a singlemode optical fiber to a very thin thickness maintaining the taper condition to keep adiabatically the single-mode condition [19]. Due to tapering, the original core is almost vanishing. Therefore, the refractive indices that determine the guiding properties of the tapered fiber are the refractive index of the original silica clad and the refractive index of the surrounding vacuum. The refractive index and the radius of the tapered silica clad will be henceforth referred to simply as the fiber refractive index $n_{1}$ and the fiber radius $a$, respectively.

\section{A. Two-color optical potential}

Consider an atom moving in a potential $U$ outside the fiber. If $U$ has a local minimum at a point outside the fiber, the atom can be trapped in the vicinity of this point. Our task is to create a potential with a trapping minimum sufficiently far from the fiber surface to make the effects of surface interaction and heating negligible. For this purpose, we use two light fields in the fundamental modes 1 and 2 with differing frequencies $\omega_{1}$ and $\omega_{2}$, respectively (wavelengths $\lambda_{1}$ and $\lambda_{2}$, respectively, and free-space wave numbers $k_{1}$ and $k_{2}$, respectively). A schematic of our design is shown in Fig. 1] Assume that the single-mode condition [20] $V_{i} \equiv k_{i} a \sqrt{n_{1}^{2}\left(\omega_{i}\right)-n_{2}^{2}}<V_{c} \cong 2.405$ is satisfied for both modes $(i=1$ or 2$)$. In addition, assume that the input fields are circularly polarized. In this case, the polarization of the transverse component of each propagating field rotates elliptically in time, the orbit rotates circu-

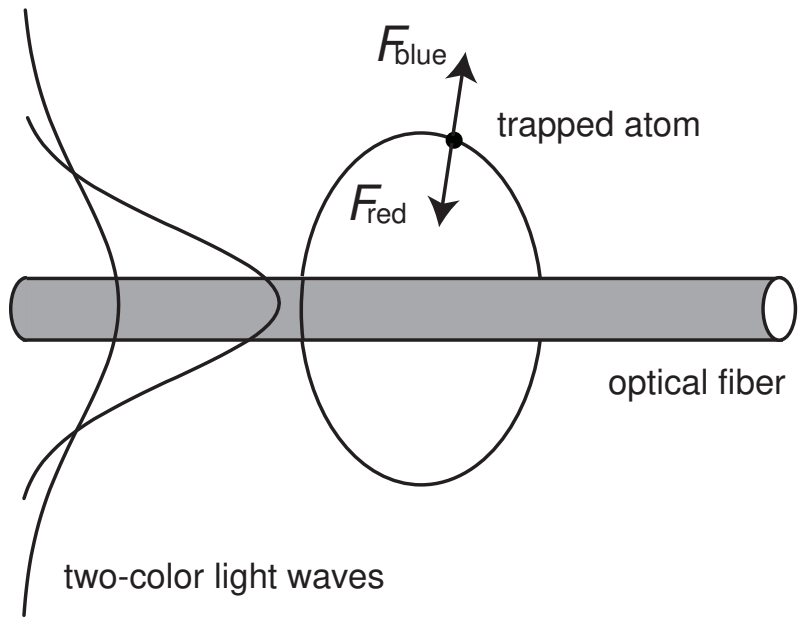

FIG. 1: Schematic of atom trapping and guiding around an optical fiber.

larly in space, and the spatial distribution of the field intensity is cylindrically symmetric [18]. Outside the fiber, in the cylindrical coordinates $\{r, \varphi, z\}$, the time-averaged intensity of the electric field in mode $i$ is given by [18, 20]

$$
\left|E_{i}\right|^{2}=\mathcal{E}_{i}^{2}\left[K_{0}^{2}\left(q_{i} r\right)+w_{i} K_{1}^{2}\left(q_{i} r\right)+f_{i} K_{2}^{2}\left(q_{i} r\right)\right] .
$$

Here the notation $K_{n}$ stands for the modified Bessel functions of the second kind, $q_{i}$ characterizes the decay of the field outside the fiber, $w_{i}$ and $f_{i}$ describe the deviations of the exact fundamental mode $\mathrm{HE}_{11}$ from the approximate mode $\mathrm{LP}_{01}$, and $\mathcal{E}_{i}$ determines the strength of the electric field. The coefficients $w_{i}$ and $f_{i}$ are defined as $w_{i}=2 q_{i}^{2} / \beta_{i}^{2}\left(1-s_{i}\right)^{2}$ and $f_{i}=\left(1+s_{i}\right)^{2} /\left(1-s_{i}\right)^{2}$, where $s_{i}=\left(1 / q_{i}^{2} a^{2}+1 / h_{i}^{2} a^{2}\right) /\left[J_{1}^{\prime}\left(h_{i} a\right) / h_{i} a J_{1}\left(h_{i} a\right)+\right.$ $\left.K_{1}^{\prime}\left(q_{i} a\right) / q_{i} a K_{1}\left(q_{i} a\right)\right]$. The parameters $q_{i}$ and $h_{i}$ are related to the longitudinal propagation constant $\beta_{i}$ as $q_{i}=\left(\beta_{i}^{2}-n_{2}^{2} k_{i}^{2}\right)^{1 / 2}$ and $h_{i}=\left[n_{1}^{2}\left(\omega_{i}\right) k_{i}^{2}-\beta_{i}^{2}\right]^{1 / 2}$. The parameter $\beta_{i}$ is determined by the eigenvalue equation for the fundamental mode at the frequency $\omega_{i}[20]$.

We assume that the atom is in the ground state and the fields are off resonance with the atom. The optical potential of the atom in the field of mode $i$ is then given by [21]

$$
U_{i}=-\frac{1}{4} \alpha_{i}\left|E_{i}\right|^{2},
$$

where $\alpha_{i}=\alpha\left(\omega_{i}\right)$ is the real part of the atomic polarizability at the optical frequency $\omega_{i}$. The factor $1 / 4$ in Eq. (2) results from the fact that the dipole of the atom is not a permanent dipole but is induced by the field, giving $1 / 2$, and from the fact that the intensity is averaged over optical oscillations, giving another $1 / 2$.

In general, the function $\alpha(\omega)$ for a ground-state atom is given by 21] $\alpha(\omega)=\left(e^{2} / m_{e}\right) \sum_{j} f_{a j}\left(\omega_{j a}^{2}-\omega^{2}\right) /\left[\left(\omega_{j a}^{2}-\right.\right.$ $\left.\left.\omega^{2}\right)^{2}+\gamma_{j a}^{2} \omega^{2}\right]$. Here $e$ and $m_{e}$ are the electric charge 
and mass, respectively, of the electron, and $\omega_{j a}, f_{a j}$, and $\gamma_{j a}$ are the frequency, absorption oscillator strength, and emission transition probability, respectively, of the spectral line $j a$. When we use the relation $\gamma_{j a}=$ $f_{a j} e^{2} \omega_{j a}^{2} g_{a} / 2 \pi m_{e} \epsilon_{0} c^{3} g_{j}$, we find

$$
\alpha(\omega)=2 \pi \epsilon_{0} c^{3} \sum_{j} \frac{g_{j}}{g_{a}} \frac{\gamma_{j a}\left(1-\omega^{2} / \omega_{j a}^{2}\right)}{\left(\omega_{j a}^{2}-\omega^{2}\right)^{2}+\gamma_{j a}^{2} \omega^{2}} .
$$

Here $g_{j}$ is the statistical weight of the excited level $|j\rangle$, and $g_{a}$ is the statistical weight of the ground-state manifold $|a\rangle$.

We note that, in the model of a two-level atom, the real part of the polarizability can be approximated as $\alpha=-\pi \epsilon_{0} c^{3} \gamma_{b a} / \omega_{b a}^{3} \Delta=-d^{2} / \hbar \Delta$. Here $\Delta=\omega-\omega_{b a}$ is the detuning of the optical frequency $\omega$ from the atomic frequency $\omega_{b a}$ and $d$ is the projection of the dipole moment onto an axis. In deriving the above approximation for $\alpha$, it has been assumed that $\Delta$ is large compared to the linewidth $\gamma_{b a}$ but is small compared to the optical and atomic frequencies. The corresponding approximate expression for the optical potential of the atom is $U_{0}=\hbar \Omega^{2} / \Delta$ where $\Omega=d|E| / 2 \hbar$ is the Rabi frequency. When the field frequency $\omega$ is far from resonance with the atomic frequencies $\omega_{j a}$, we must take into account the multilevel structure of the atom.

Assume that the timescale of atomic motion is much slower than the beating period of the two light fields, that is, the inverse of their frequency difference. Then, the optical potentials of the two fields add up to give the net optical potential

$$
U=U_{1}+U_{2} .
$$

The sign of the optical potential of each mode is controlled by the sign of the mode detuning. We choose a red detuning $\left(\Delta_{1}<0\right)$ for the field in mode 1 and a blue detuning $\left(\Delta_{2}>0\right)$ for the field in mode 2. This allows both attractive (red-detuned) and repulsive (bluedetuned) potentials to be created. When we substitute Eq. (1) into Eq. (2) and then the result into Eq. (4), we obtain

$$
\begin{aligned}
U(r)= & G_{2}\left[K_{0}^{2}\left(q_{2} r\right)+w_{2} K_{1}^{2}\left(q_{2} r\right)+f_{2} K_{2}^{2}\left(q_{2} r\right)\right] \\
& -G_{1}\left[K_{0}^{2}\left(q_{1} r\right)+w_{1} K_{1}^{2}\left(q_{1} r\right)+f_{1} K_{2}^{2}\left(q_{1} r\right)\right] .
\end{aligned}
$$

Here $G_{1}=\alpha_{1} \mathcal{E}_{1}^{2} / 4=\left|\alpha_{1}\right| \mathcal{E}_{1}^{2} / 4$ and $G_{2}=-\alpha_{2} \mathcal{E}_{2}^{2} / 4=$ $\left|\alpha_{2}\right| \mathcal{E}_{2}^{2} / 4$ are positive coupling parameters. They are proportional to the powers of the corresponding light fields. Since $\omega_{1}<\omega_{2}$, we have $q_{1}<q_{2}$, that is, the evanescent decay length $\Lambda_{1}=1 / q_{1}$ of the red-detuned field is larger than the evanescent decay length $\Lambda_{2}=1 / q_{2}$ of the blue-detuned field [8, 20]. When the intensity of the bluedetuned field is large enough, the net optical potential $U$ is repulsive at short range and attractive at long range [9]. Such a potential possesses a local minimum point, where the two forces cancel each other, leading to the possibility of atom trapping. Since $U$ is independent of $\varphi$ and $z$, a minimum point in the one-dimensional space $\{r\}$ corresponds to a cylindrical shell of minimum points in the three-dimensional space $\{r, \varphi, z\}$.

To get insight into the trapping properties of the net optical potential $U$, we perform an analytical treatment. To simplify this treatment, we assume that, from one side, the coefficients $w_{i}$ and $f_{i}$ are sufficiently small and, from another side, the parameters $q_{i} a$ are sufficiently large that the contributions of the terms containing $w_{i}$ and $f_{i}$ in Eq. (5) are not substantial [18. Then, we obtain the following approximate expression:

$$
U(r)=G_{2} K_{0}^{2}\left(q_{2} r\right)-G_{1} K_{0}^{2}\left(q_{1} r\right) .
$$

We use this approximation only for our analysis but not for our numerical calculations.

We introduce the notation $r_{m}$ and $U_{m}=U\left(r_{m}\right)$ for the local minimum point and local minimum value of $U$, respectively. From $U^{\prime}\left(r_{m}\right)=0$, we find

$$
F\left(r_{m}\right)=A,
$$

where

$$
F(r)=\frac{K_{0}\left(q_{1} r\right) K_{1}\left(q_{1} r\right)}{K_{0}\left(q_{2} r\right) K_{1}\left(q_{2} r\right)}
$$

and

$$
A=\frac{q_{2} G_{2}}{q_{1} G_{1}}
$$

Since $q_{1}<q_{2}$, the function $F(r)$ is a monotonically increasing function of $r$. Hence, Eq. (7) has a solution $r_{m}>a$ if $A>F(a)$, that is, if

$$
\frac{G_{2}}{G_{1}}>\frac{q_{1} K_{0}\left(q_{1} a\right) K_{1}\left(q_{1} a\right)}{q_{2} K_{0}\left(q_{2} a\right) K_{1}\left(q_{2} a\right)} .
$$

Condition (10) means that there exists a trapping minimum outside the fiber if the intensity of the blue-detuned light is large enough or if the intensity of the red-detuned light is small enough (but not zero). Note that, when the power of the blue-detuned light increases or the power of the red-detuned light decreases, the depth $U_{D} \equiv-U_{m}=$ $\left|U_{m}\right|$ of the trapping potential decreases and the local minimum point $r_{m}$ is shifted farther away from the fiber surface. Oppositely, when the power of the blue-detuned light decreases or the power of the red-detuned light increases, the potential depth increases and the local minimum point is shifted towards the fiber surface.

The atom can be prevented from coming too close to the fiber surface before being loaded into the trap if the net optical potential achieves a non-negative value at the fiber surface, that is, if $U(a) \geq 0$. This requirement will be fulfilled if

$$
\frac{G_{2}}{G_{1}} \geq \frac{K_{0}^{2}\left(q_{1} a\right)}{K_{0}^{2}\left(q_{2} a\right)} .
$$


When condition (11) is satisfied, condition (10) is also satisfied. To maximize the depth of the trapping minimum, we optimize the powers of the two field in such a way that condition (11) reduces to an equality, namely,

$$
\frac{G_{2}}{G_{1}}=\frac{K_{0}^{2}\left(q_{1} a\right)}{K_{0}^{2}\left(q_{2} a\right)} .
$$

Then, Eq. (6) yields

$$
U(r)=G_{0}\left[\frac{K_{0}^{2}\left(q_{2} r\right)}{K_{0}^{2}\left(q_{2} a\right)}-\frac{K_{0}^{2}\left(q_{1} r\right)}{K_{0}^{2}\left(q_{1} a\right)}\right],
$$

where $G_{0}=G_{1} K_{0}^{2}\left(q_{1} a\right)=G_{2} K_{0}^{2}\left(q_{2} a\right)$.

We analyze the effects of the evanescent decay parameters $q_{1}$ and $q_{2}$ on the trapping potential under condition (12). For this purpose, we fix $q_{1}$ and $G_{1}$ (or $q_{2}$ and $G_{2}$ ) and increase $q_{2}$ and $G_{2}$ (or decrease $q_{1}$ and $G_{1}$ ) in such a way that equality (12) is kept. This leads to a reduction in $U$ and, consequently, to a decrease in the minimum value $U_{m}$, that is, to an increase in the depth $U_{D}=-U_{m}$ of the trapping potential. Such variations also make the minimum point of the potential closer to the fiber surface. Note that, when the fiber radius is small, the evanescent decay length $\Lambda$ is a fast increasing function of the light wavelength $\lambda[8]$. Therefore, using a thin fiber, we can obtain a large ratio of $q_{2}$ to $q_{1}$ by choosing a small $\lambda_{2}$ and/or a large $\lambda_{1}$. Thus, a reduction in the blue-detuned light wavelength $\lambda_{2}$ or an increase in the red-detuned light wavelength $\lambda_{1}$ allows us to increase the potential depth provided the power of the light field $E_{2}$ or $E_{1}$, respectively, can be optimized appropriately to satisfy condition (12). However, $\lambda_{2}$ must not be too small because it is limited by the single-mode condition. In addition, $\lambda_{1}$ must not be too large because a larger wavelength $\lambda_{1}$ leads to a larger penetration length $\Lambda_{1}$ and, therefore, to a smaller potential magnitude for the same power of light. Furthermore, the detunings $\Delta_{i}$ of the light fields from the atomic resonance frequency must not be too large because a larger detuning leads to a smaller polarizability and, consequently, to a smaller potential magnitude for the same power of light.

In general, the polarizability of an atom is a complex characteristic. The imaginary part of the polarizability is given by [21]

$$
\kappa(\omega)=2 \pi \epsilon_{0} c^{3} \sum_{j} \frac{g_{j}}{g_{a}} \frac{\gamma_{j a}^{2} \omega / \omega_{j a}^{2}}{\left(\omega_{j a}^{2}-\omega^{2}\right)^{2}+\gamma_{j a}^{2} \omega^{2}} .
$$

It is responsible for spontaneous scattering. The rate of spontaneous scattering caused by a single light field $E_{i}$ is given by

$$
\Gamma_{i}^{(\mathrm{sc})}=\frac{1}{4 \hbar} \kappa_{i}\left|E_{i}\right|^{2},
$$

where $\kappa_{i}=\kappa\left(\omega_{i}\right)$. Spontaneous scattering limits the coherence time of the trap. For atoms spending time close to the minimum $r_{m}$ of the two-color potential $U$, the net scattering rate is

$$
\Gamma_{\mathrm{sc}}=\Gamma_{1}^{(\mathrm{sc})}\left(r_{m}\right)+\Gamma_{2}^{(\mathrm{sc})}\left(r_{m}\right)
$$

and the characteristic coherence time is 11,12

$$
\tau_{\mathrm{coh}}=\frac{1}{\Gamma_{\mathrm{sc}}}
$$

Every scattered photon imparts a recoil energy $\theta_{i}^{(\text {rec })}=$ $\left(\hbar k_{i}\right)^{2} / 2 M$ to the atom, where $M$ is the mass of the atom. Therefore, the absorption of mode photons and emission of other photons result in a loss of atoms from the trapping potential. For a trap depth $U_{D}$, the quantity 12

$$
\tau_{\text {trap }}=\frac{U_{D}}{2 \sum_{i} \theta_{i}^{(\mathrm{rec})} \Gamma_{i}^{(\mathrm{sc})}\left(r_{m}\right)}
$$

characterizes the trap lifetime due to recoil heating. When the light field frequencies are near to the atomic resonances, the scattering rates are large and, therefore, the coherence time and the trap lifetime are small. To produce a trap with a large coherence time and a large trap lifetime, we must use far-off-resonance fields.

\section{B. van der Waals potential}

An atom near the surface of a medium undergoes a van der Waals force. The van der Waals potential of an atom near the surface of a cylindrical dielectric rod is given by [22]

$$
\begin{aligned}
V(r)= & \frac{\hbar}{4 \pi^{3} \epsilon_{0}} \sum_{n=-\infty}^{\infty} \int_{0}^{\infty} d k\left[k^{2} K_{n}^{\prime 2}(k r)\right. \\
& \left.+\left(k^{2}+n^{2} / r^{2}\right) K_{n}^{2}(k r)\right] \int_{0}^{\infty} d \xi \alpha(i \xi) G_{n}(i \xi),
\end{aligned}
$$

where

$$
G_{n}(\omega)=\frac{\left[\epsilon(\omega)-\epsilon_{0}\right] I_{n}(k a) I_{n}^{\prime}(k a)}{\epsilon_{0} I_{n}(k a) K_{n}^{\prime}(k a)-\epsilon(\omega) I_{n}^{\prime}(k a) K_{n}(k a)} .
$$

Here $\epsilon(\omega)$ is the dynamical dielectric function and $I_{n}$ is the modified Bessel function of the first kind.

We calculate the van der Waals potential of a groundstate cesium atom near a silica fiber surface. The dynamical dielectric function of silica is given by 23 . $\frac{\epsilon(\omega)}{\epsilon_{0}}=1+\frac{0.6961663 \lambda^{2}}{\lambda^{2}-0.0684043^{2}}+\frac{0.4079426 \lambda^{2}}{\lambda^{2}-0.1162414^{2}}+\frac{0.8974794 \lambda^{2}}{\lambda^{2}-9.896161^{2}}$, where $\lambda$ is in the units of $\mu \mathrm{m}$. To calculate the integral (19), we use the approximate expression $\alpha(\omega)=$ $2 \pi \epsilon_{0} c^{3} \sum_{j} g_{j} \gamma_{j a} /\left[g_{a} \omega_{j a}^{2}\left(\omega_{j a}^{2}-\omega^{2}\right)\right]$. This approximation is justified because the resonant frequencies of the ground-state cesium atom are substantially different from the resonant frequencies of silica. We take into account four dominant lines of the atom, namely, $\lambda_{1 a}=$ 


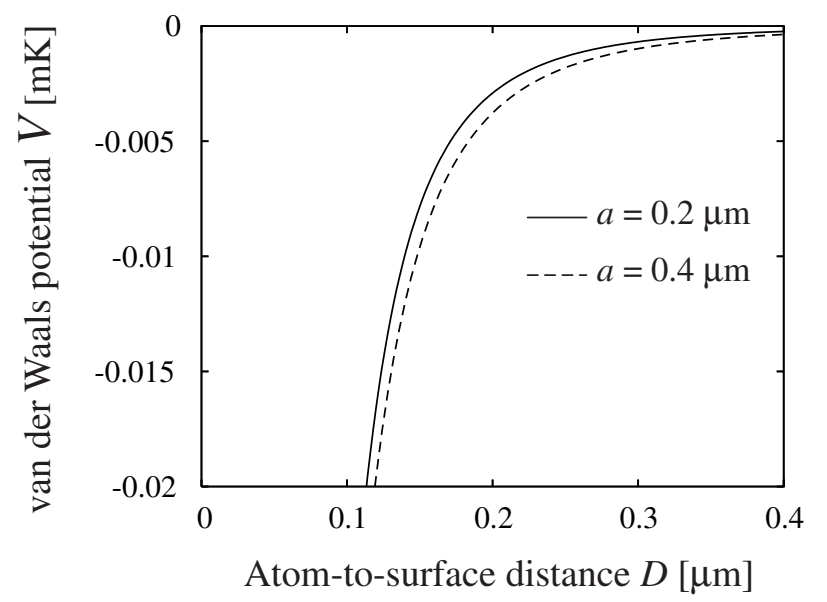

FIG. 2: van der Waals potential $V$ of a ground-state cesium atom near a thin cylindrical silica fiber.

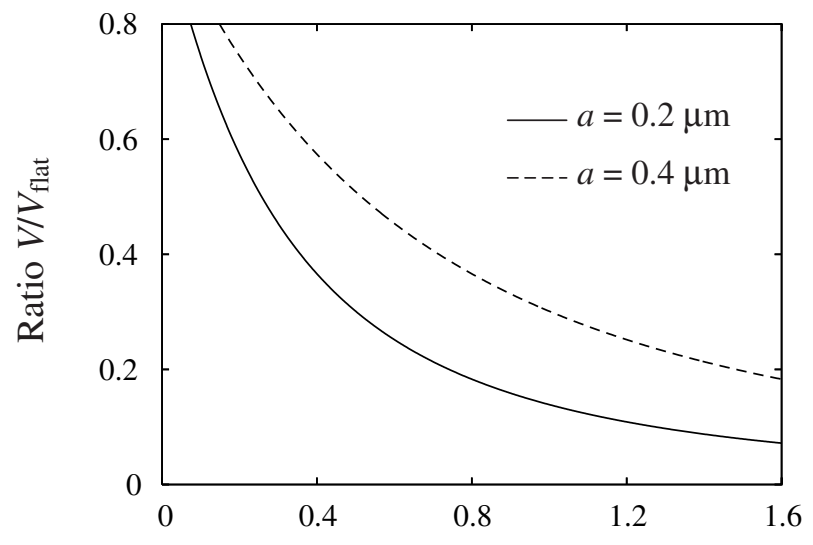

Atom-to-surface distance $D[\mu \mathrm{m}]$

FIG. 3: Ratio between the van der Waals potentials $V$ and $V_{\text {flat }}$ of a ground-state cesium atom near a thin cylindrical silica fiber and a flat-surface bulk silica dielectric, respectively.

$852.113 \mathrm{~nm}, \lambda_{2 a}=894.347 \mathrm{~nm}, \lambda_{3 a}=455.528 \mathrm{~nm}$, and $\lambda_{4 a}=459.317 \mathrm{~nm}$, see [25]. The emission transition probabilities of these lines are $\gamma_{1 a}=3.276 \times 10^{7}$ $\mathrm{s}^{-1}, \gamma_{2 a}=2.87 \times 10^{7} \mathrm{~s}^{-1}, \gamma_{3 a}=1.88 \times 10^{6} \mathrm{~s}^{-1}$, and $\gamma_{4 a}=8 \times 10^{5} \mathrm{~s}^{-1}$. The statistical weights of the four corresponding upper states are $g_{1}=4, g_{2}=2, g_{3}=4$, and $g_{4}=2$. The statistical weight of the ground state is $g_{a}=2$.

In Fig. 2 we plot the van der Waals potential $V$ of a ground-state cesium atom near a cylindrical silica fiber as a function of the atom-to-surface distance $D=r-a$. The comparison between the solid line $(a=0.2 \mu \mathrm{m})$ and the dashed line $(a=0.4 \mu \mathrm{m})$ shows that a smaller fiber radius $a$ leads to a smaller magnitude and a less steep slope of the van der Waals potential $V$.

When the fiber radius $a$ is small compared to the atom-to-surface distance $D$, the van der Waals poten- tial $V$ is, in general, different from the flat-surface bulk-medium van der Waals potential $V_{\text {flat }}=-C_{3} / D^{3}$. Here the coefficient $C_{3}$ is determined by [24] $C_{3}=$ $\left(\hbar / 16 \pi^{2} \epsilon_{0}\right) \int_{0}^{\infty} d \xi \alpha(i \xi)\left[\epsilon(i \xi)-\epsilon_{0}\right] /\left[\epsilon(i \xi)+\epsilon_{0}\right]$. For cesium atoms and flat silica surfaces, this coefficient is estimated to be $C_{3} \cong 5.6 \times 10^{-49} \mathrm{~J} \mathrm{~m}^{3} \cong 4.1 \times 10^{-5} \mathrm{mK} \mu \mathrm{m}^{3}$. Figure 3 illustrates the difference between the fiber-surface potential $V$ and the flat-surface potential $V_{\text {flat }}$. The figure shows that $V / V_{\text {flat }}<1$, that is, the magnitude of $V$ is smaller than the magnitude of $V_{\text {flat }}$. When the atom-tosurface distance $D$ tends to zero, the ratio $V / V_{\text {flat }}$ tends to unity, that is, the two potentials $V$ and $V_{\text {flat }}$ tend to become the same. When $D$ increases, the ratio $V / V_{\text {flat }}$ reduces, that is, the relative difference between $V$ and $V_{\text {flat }}$ increases.

\section{Total potential}

The total potential $U_{\text {tot }}$ of the atom is the sum of the net optical potential $U$ and the van der Waals potential $V$, i.e.,

$$
U_{\text {tot }}=U+V \text {. }
$$

We use Eqs. (5), (19), and (21) to calculate the total potential $U_{\text {tot }}$ of a ground-state cesium atom outside a thin cylindrical silica fiber with two circularly polarized input light fields. The ground-state cesium atom has two strong transitions, at $852 \mathrm{~nm}\left(D_{2}\right.$ line $)$ and $894 \mathrm{~nm}\left(D_{1}\right.$ line). To trap the atom, we use red- and blue-detuned lights with wavelengths $\lambda_{1}=1.06 \mu \mathrm{m}$ and $\lambda_{2}=700$ $\mathrm{nm}$, respectively. The detunings of the lights from the dominant $D_{2}$ line of the atom are $\Delta_{1} / 2 \pi=-69 \mathrm{THz}$ and $\Delta_{2} / 2 \pi=76 \mathrm{THz}$. The fiber radius is $a=0.2 \mu \mathrm{m}$. This radius is small enough to create a large ratio of $q_{2}$ to $q_{1}$ and to reduce the effect of the van der Waals force [8]. For the above parameters, we find $q_{1} a \cong 0.2438$ and $q_{2} a \cong 0.9686$. The corresponding evanescent decay lengths are $\Lambda_{1} \cong 0.8 \mu \mathrm{m}$ and $\Lambda_{2} \cong 0.2 \mu \mathrm{m}$. The relative difference between the decay lengths is measured by the parameter $\alpha_{\Lambda}=\left(\Lambda_{1}-\Lambda_{2}\right) / \Lambda_{2} \cong 3$. The obtained value of this parameter is much larger than the characteristic value $\alpha_{\Lambda} \cong 0.47$ estimated for the case of channel guides with the TE and TM modes [1].

In Fig. 14 we plot the contributions to the total trapping potential $U_{\text {tot }}$ of the atom. The figure shows that the evanescent decay length of the red-detuned light is substantially larger than that of the blue-detuned light. The net optical potential $U$ has a local minimum value $U_{m} \cong-2.9 \mathrm{mK}$ at $r_{m} \cong 0.37 \mu \mathrm{m}$, well outside the fiber. The van der Waals potential is only about $-5 \mu \mathrm{K}$ at the distance $r_{m}-a \cong 0.17 \mu \mathrm{m}$ from the fiber surface and makes just a small contribution in the vicinity of the trapping minimum. It is interesting to note that the depth $U_{D} \cong 2.9 \mathrm{mK}$ of the net optical potential is comparable to the red-detuned potential and is larger than the blue-detuned potential at the trapping point. These characteristics of the two-color fiber scheme are much 


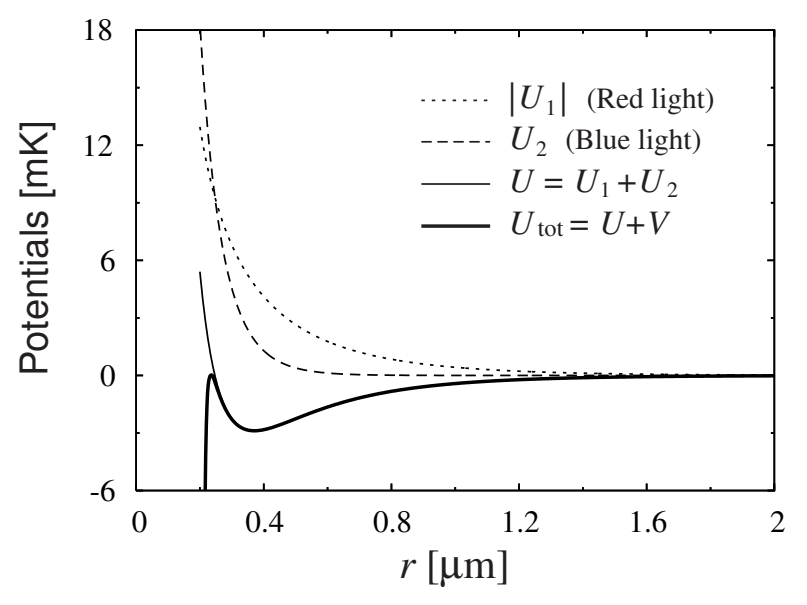

FIG. 4: Contributions to the total trapping potential $U_{\text {tot }}$ of a ground-state cesium atom outside a vacuum-clad subwavelength silica fiber with two circularly polarized input light fields. The radius of the fiber is $a=0.2 \mu \mathrm{m}$. The absolute value of the red-detuned component $U_{1}$ (dotted line) subtracts from the blue-detuned component $U_{2}$ (dashed line) to give the net optical potential $U$ (thin solid line). This is modified by the van der Waals surface interaction, giving the total potential $U_{\text {tot }}$ (thick solid line). The light wavelengths are $\lambda_{1}=1.06$ $\mu \mathrm{m}$ and $\lambda_{2}=700 \mathrm{~nm}$. The light powers are $P_{1}=30 \mathrm{~mW}$ and $P_{2}=29 \mathrm{~mW}$.

better than those of the two-color integrated-waveguide scheme, where the red- and blue-detuned potentials are large but the net potential is small [12].

We estimate some critical trapping parameters for the case of Fig. 4 We find that the rates of scattering due to the trapping fields at the potential minimum are $\Gamma_{1}^{(\mathrm{sc})} \cong 22.39 \mathrm{~s}^{-1}$ and $\Gamma_{2}^{(\mathrm{sc})} \cong 8.46 \mathrm{~s}^{-1}$. Accordingly, the net scattering rate and the coherence time are $\Gamma_{\mathrm{sc}} \cong 30.85 \mathrm{~s}^{-1}$ and $\tau_{\text {coh }} \cong 32 \mathrm{~ms}$, respectively. Since the recoil energies due to single red- and blue-detuned photons are $\theta_{1}^{\text {(rec) }} \cong 0.06 \mu \mathrm{K}$ and $\theta_{2}^{(\mathrm{rec})} \cong 0.15 \mu \mathrm{K}$, respectively, the trapping lifetime due to recoil heating is estimated to be $\tau_{\text {trap }} \cong 541 \mathrm{~s}$. At the trapping minimum, the radial oscillation frequency is $\omega_{r} / 2 \pi \cong 492 \mathrm{KHz}$, giving an estimate of about $23.6 \mu \mathrm{K}$ for the energy of the atomic mode spacing.

Due to the circular polarization of the input fields, $U_{\text {tot }}$ is cylindrically symmetric. In Fig. 囵 we plot the spatial profile of $U_{\text {tot }}$ in the fiber transverse plane. The figure shows that $U_{\text {tot }}$ is independent of the azimuthal angle $\varphi$ and has a set of deep minima formed as a ring surrounding the fiber. Since the atom can move freely along the direction $z$ of the fiber axis, $U_{\text {tot }}$ is also independent of $z$. Therefore, the ring of the potential minima in the transverse plane results in a cylindrical shell of the minima of the trapping potential in the three-dimensional space.

Figure [ illustrates the effect of the power $P_{1}$ of the red-detuned light on the total potential $U_{\text {tot. }}$. As seen, an increase in $P_{1}$ leads to an increase in the depth $U_{D}$ of the

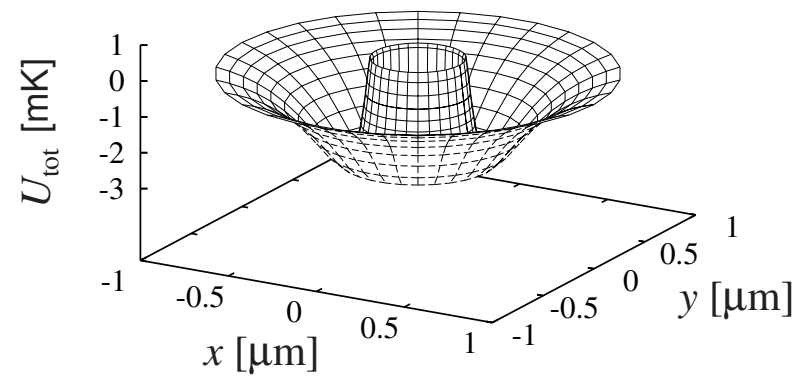

FIG. 5: Transverse-plane profile of the total potential $U_{\text {tot }}$ produced by lights that are circularly polarized at the input. All the parameters are the same as for Fig. 4.

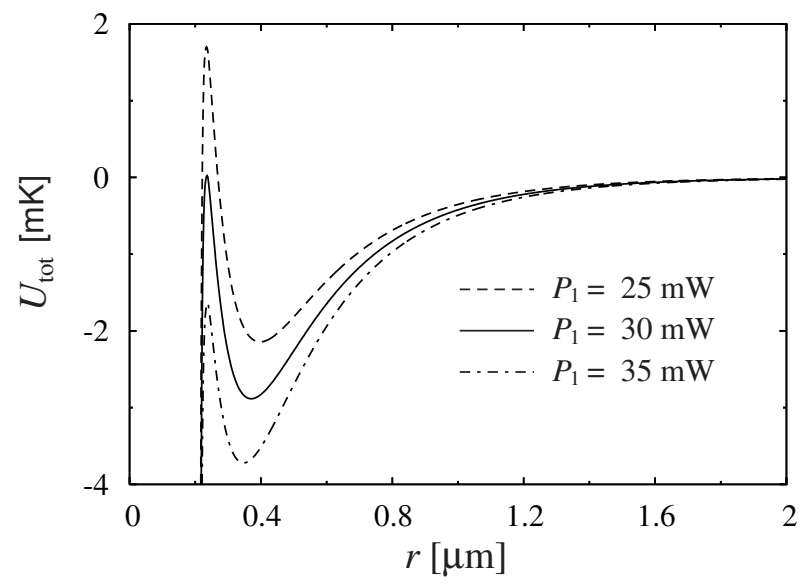

FIG. 6: Effect of the power $P_{1}$ of the red-detuned light on the total potential $U_{\text {tot }}$. The power of the blue-detuned light is fixed at $P_{2}=29 \mathrm{~mW}$. All the other parameters are the same as for Fig. 4

trapping potential and to a shift of the local minimum point $r_{m}$ towards the fiber surface. However, the increase in $P_{1}$ also reduces the height of the repulsive wall in the region of $r<r_{m}$.

Figure 7 illustrates the effect of the power $P_{2}$ of the blue-detuned light on the total potential $U_{\text {tot }}$. As seen, an increase in $P_{2}$ leads to a decrease in the depth $U_{D}$ of the trapping potential and to a shift of the local minimum point $r_{m}$ farther away from the fiber surface. Meanwhile, the height of the repulsive wall in the region of $r<r_{m}$ is increased. The comparison between Figs. [6] and [7] show that the powers $P_{1}$ and $P_{2}$ of the red- and blue-detuned lights, respectively, have opposite effects on the total potential of the atom. Therefore, to produce a potential with a deep trapping minimum outside the fiber and with a high repulsive wall in the region of $r<r_{m}$, the ratio between $P_{1}$ and $P_{2}$ must be optimized appropriately.

Due to the cylindrical symmetry of the total potential $U_{\text {tot }}$, the component $L_{z}$ of the angular momentum of the atom is conserved. In the eigenstate problem, we have $L_{z}=\hbar m$, where $m$ is an integer, called the ro- 


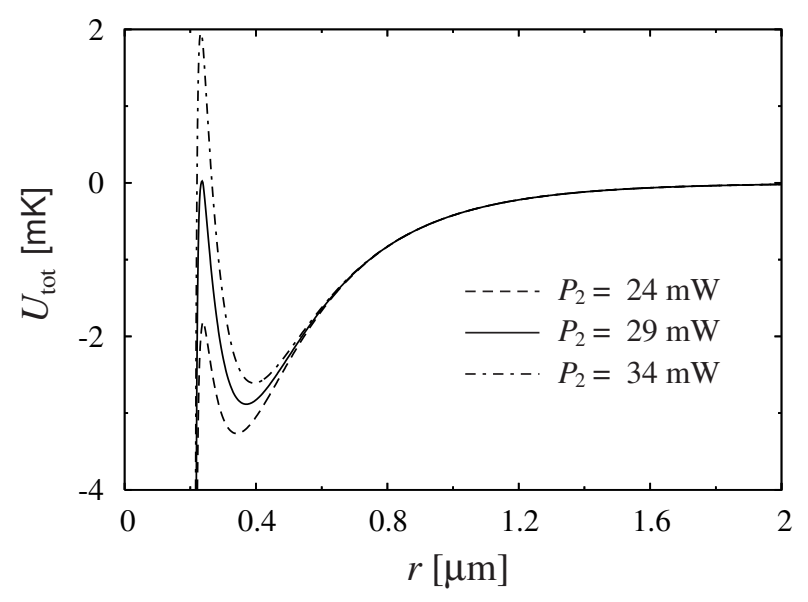

FIG. 7: Effect of the power $P_{2}$ of the blue-detuned light on the total potential $U_{\text {tot }}$. The power of the red-detuned light is fixed at $P_{1}=30 \mathrm{~mW}$. All the other parameters are the same as for Fig. 4

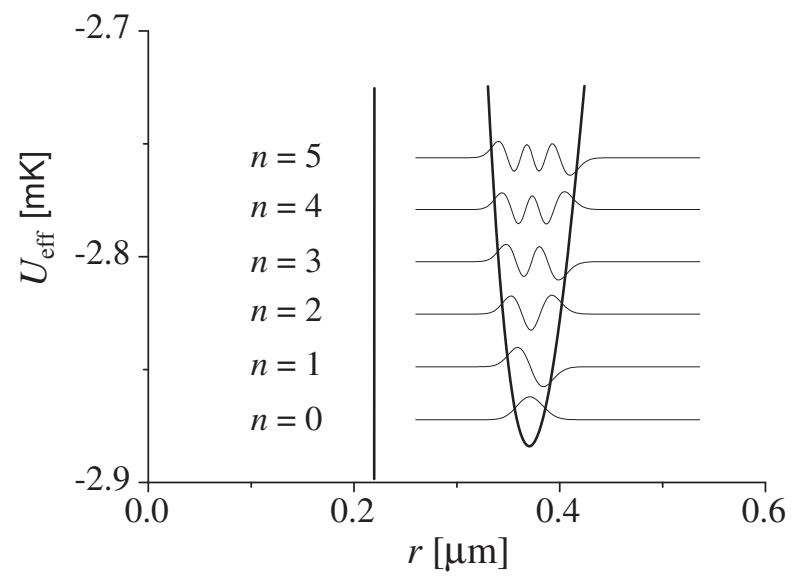

FIG. 8: Bound states for the first six levels $(n=0,1,2,3,4$, and 5) of the radial motion of a cesium atom in the effective potential $U_{\text {eff }}=U_{\text {tot }}+\hbar^{2}\left(m^{2}-1 / 4\right) / 2 M r^{2}$. The rotational quantum number is $m=0$. All the other parameters are the same as for Fig. 4

tational quantum number. The centrifugal potential of the atom is given by $U_{\text {cf }}=\hbar^{2}\left(m^{2}-1 / 4\right) / 2 M r^{2}$. The radial motion of the atom can be treated as the onedimensional motion of a particle in the effective potential $U_{\text {eff }}=U_{\text {tot }}+U_{\text {cf. }}$. In Fig. 8 we plot the eigenfunctions for the first six levels of the radial motion of the atom in the effective potential $U_{\text {eff }}$ with the rotational quantum number $m=0$. The energy of the ground state is $E_{0} \cong-2.872 \mathrm{mK}$. The spacing between the the energies of the ground state and the first excited state is roughly $23 \mu \mathrm{K}$. The characteristic size of the ground state is $\Delta r \cong 8.8 \mathrm{~nm}$.

\section{TWO-COLOR TRAP WITH LINEARLY POLARIZED INPUT LIGHTS}

The formation of a set of trapping minima as a ring in the fiber transverse plane (or as a shell in the threedimensional space), shown in the previous section, is due to the cylindrical symmetry of all of the components of the total potential. In order to confine atoms to vicinities of single local points in the fiber transverse plane, we need to break the symmetry. An asymmetric optical potential can be obtained when one or both trapping light fields are linearly polarized at the input. Indeed, it has been shown that, due to the thin thickness of the fiber and the high contrast between the refractive indices of the silica core and the vacuum clad, the intensity distribution of the field in a fundamental mode with quasi-linear polarization strongly depends on the azimuthal angle, especially in the vicinity of the fiber surface [18].

Regarding the properties of the trapping potential, there is no substantial qualitative difference between the case where both input fields are linearly polarized and the case where one is linearly polarized and the other one is circularly polarized. Therefore, we consider here, as an example, the case where both input fields are linearly polarized, along the same direction, namely, the $x$ direction. In this case, the polarization of each light field propagating along the fiber is quasi-linear and the spatial distribution of the field intensity is not cylindrically symmetric 18. Outside the fiber, the time-averaged intensity of the electric field in mode $i$ is given by [18, 20]

$$
\begin{aligned}
\left|E_{i}\right|^{2}= & \mathcal{E}_{i}^{2}\left\{K_{0}^{2}\left(q_{i} r\right)+w_{i} K_{1}^{2}\left(q_{i} r\right)+f_{i} K_{2}^{2}\left(q_{i} r\right)\right. \\
& \left.+\left[w_{i} K_{1}^{2}\left(q_{i} r\right)+\xi_{i} K_{0}\left(q_{i} r\right) K_{2}\left(q_{i} r\right)\right] \cos 2 \varphi\right\} .
\end{aligned}
$$

Here $\xi_{i}=2\left(1+s_{i}\right) /\left(1-s_{i}\right)$. We use the above field intensity distributions and Eq. (21) to calculate the optical potentials $U_{1}$ and $U_{2}$ of an atom in the red- and bluedetuned fields. The net optical potential $U=U_{1}+U_{2}$ is then found to be

$$
\begin{aligned}
U(r, \varphi)= & G_{2}\left\{K_{0}^{2}\left(q_{2} r\right)+w_{2} K_{1}^{2}\left(q_{2} r\right)+f_{2} K_{2}^{2}\left(q_{2} r\right)\right. \\
& \left.+\left[w_{2} K_{1}^{2}\left(q_{2} r\right)+\xi_{2} K_{0}\left(q_{2} r\right) K_{2}\left(q_{2} r\right)\right] \cos 2 \varphi\right\} \\
& -G_{1}\left\{K_{0}^{2}\left(q_{1} r\right)+w_{1} K_{1}^{2}\left(q_{1} r\right)+f_{1} K_{2}^{2}\left(q_{1} r\right)\right. \\
& \left.+\left[w_{1} K_{1}^{2}\left(q_{1} r\right)+\xi_{1} K_{0}\left(q_{1} r\right) K_{2}\left(q_{1} r\right)\right] \cos 2 \varphi\right\}
\end{aligned}
$$

Unlike the potential (51), the potential (23) contains additional terms that vary with the azimuthal angle $\varphi$. Due to these terms, the potential (23) is not cylindrically symmetric. Meanwhile, when the intensity of the bluedetuned field is large enough, the potential (23) is, like the potential (5), repulsive at short range and attractive at long range. For a fixed angle $\varphi$, the potential $U(r, \varphi)$ as a function of $r$ must have a local minimum value $U_{m}(\varphi)$ achieved at a point $r_{m}(\varphi)$ where the repulsive and attractive forces cancel each other. Due the cylindrical asymmetry of the potential, $U_{m}(\varphi)$ is not constant in $\varphi$. 


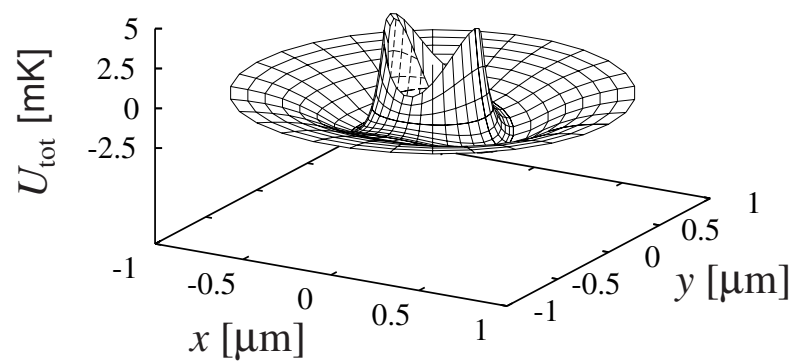

FIG. 9: Transverse-plane profile of the total potential $U_{\text {tot }}$ produced by the lights that are linearly polarized at the input. The light powers are $P_{1}=30 \mathrm{~mW}$ and $P_{2}=35 \mathrm{~mW}$. All the other parameters are the same as for Fig. 4

Consequently, $U_{m}(\varphi)$ must have a global minimum value $U_{g}=\min U_{m}(\varphi)$, which is achieved at a finite number of angles $\varphi_{m}$. Thus the potential $U(r, \varphi)$ must have a global minimum value $U_{g}$ achieved at a finite number of single points $\left(r_{m}\left(\varphi_{m}\right), \varphi_{m}\right)$ in the fiber transverse plane. Due to the axial symmetry, we have $U(r, \varphi)=U(r, \pi+\varphi)$. Therefore, there must be two minimum points for $U(r, \varphi)$ in the transverse plane. Since the atom can move freely along the direction $z$ of the fiber axis, the two minimum points in the transverse plane in fact correspond to two straight lines for the minima of the trapping potential in the three-dimensional space. These potential minimum lines can be used to trap and guide atoms along the fiber.

We use Eq. (231) as well as Eqs. (19) and (21) to calculate the total potential $U_{\text {tot }}$ of a ground-state cesium atom outside a thin cylindrical silica fiber with two linearly polarized input light fields. We plot in Fig. 9 the spatial profile of $U_{\text {tot }}$ in the fiber transverse plane. The figure shows that $U_{\text {tot }}$ varies substantially with the azimuthal angle $\varphi$, especially in the vicinity of the fiber surface. The minimum points in the radial dependence of $U_{\text {tot }}$ form a ring surrounding the fiber. Unlike the case of circular polarization, see Fig. [5] the ring at the bottom of Fig. 9 is not flat with respect to $\varphi$. This ring has two deepest points, located symmetrically on the $x$ axis (at $\varphi=0$ and $\varphi=\pi$ ). These points correspond to two straight lines, parallel to the fiber axis, for the trapping minima in the three-dimensional space.

To get a closer look at the spatial distributions of the potential along different radial directions, we replot in Fig. 10 the potential $U_{\text {tot }}$ of Fig. 9 as a function of $x$ at $y=0$ (i.e., as a function of $r$ at $\varphi=0$ ) and as a function of $y$ at $x=0$ (i.e., as a function of $r$ at $\varphi=\pi / 2$ ). Figures 10(a) and 10(b) show that, for $P_{1}=30 \mathrm{~mW}$ and $P_{2}=35 \mathrm{~mW}$, the depths of the potential minima for the $x$ and $y$ directions are about $-3.2 \mathrm{mK}$ and $-2 \mathrm{mK}$, respectively. Thus the depth of the potential minimum for the $x$ direction is deeper than that for the $y$ direction. Consequently, the local minimum for the $x$ direction is the local minimum for the whole transverse plane. The difference between the depths of the potential minima for the $x$ and $y$ directions is about $1.2 \mathrm{mK}$. Note that the

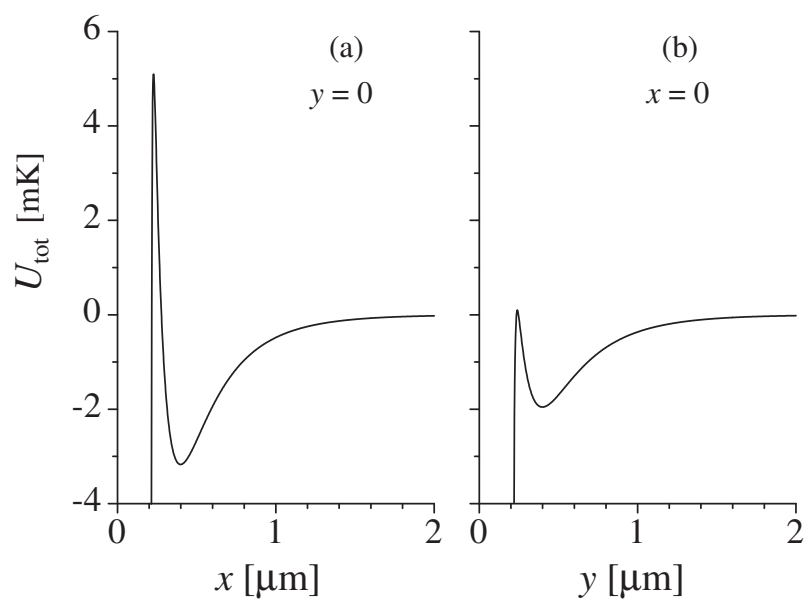

FIG. 10: Total potential $U_{\text {tot }}$ as a function of $x$ at $y=0$ (a) and as a function of $y$ at $x=0(\mathrm{~b})$. All the parameters are the same as for Fig. 9

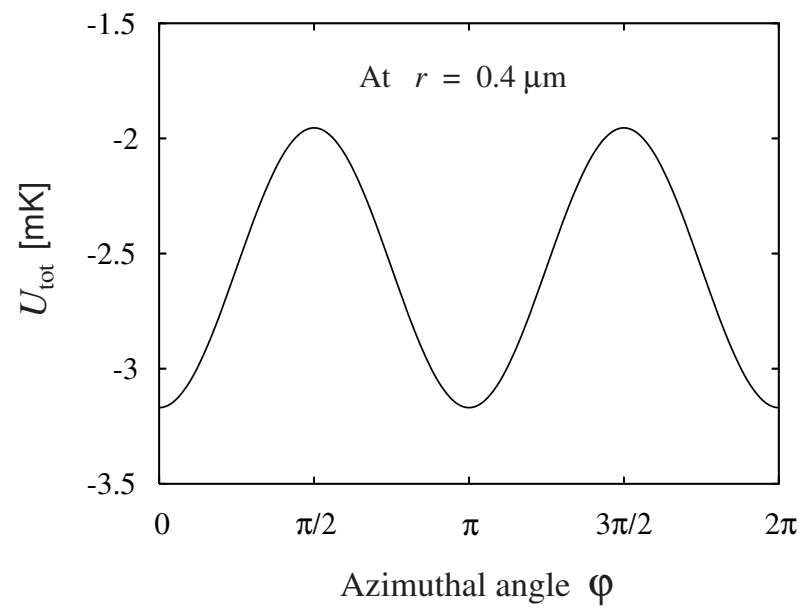

FIG. 11: Azimuthal dependence of the total potential $U_{\text {tot }}$ for $r=0.4 \mu \mathrm{m}$. All the parameters are the same as for Fig. 9

radial distances $x_{m}=r_{m}(0)$ and $y_{m}=r_{m}(\pi / 2)$ of the local minima for the $x$ and $y$ directions are almost the same, namely, $r_{m}(\varphi) \cong 0.4 \mu \mathrm{m}$.

To get insight into the variations of $U_{\text {tot }}$ along the bottom ring $r=r_{m}(\varphi) \cong 0.4 \mu \mathrm{m}$, we plot this potential in Fig. [11 as a function of $\varphi$ for a fixed radial distance $r=0.4 \mu \mathrm{m}$. As seen, along the bottom ring, $U_{\text {tot }}$ achieves its minimum value at two angles, $\varphi=0$ and $\varphi=\pi$. The difference between the maximum and minimum values of $U_{\text {tot }}$ along the bottom ring is about $1.2 \mathrm{mK}$.

We estimate some important parameters of the trap at the minimum point $\left(r=r_{m}, \varphi=0\right)$ or $\left(r=r_{m}, \varphi=\right.$ $\pi)$ in the situation of Fig. 9 We find that the rates of scattering due to the light fields at the trapping minimum are $\Gamma_{1}^{(\mathrm{sc})} \cong 25.87 \mathrm{~s}^{-1}$ and $\Gamma_{2}^{(\mathrm{sc})} \cong 10.49 \mathrm{~s}^{-1}$. Accordingly, the net scattering rate and the coherence time are $\Gamma_{\mathrm{sc}} \cong$ 
$36.36 \mathrm{~s}^{-1}$ and $\tau_{\text {coh }} \cong 27.5 \mathrm{~ms}$, respectively. The trapping lifetime due to recoil heating is estimated to be $\tau_{\text {trap }} \cong$ $500 \mathrm{~s}$. When we fit the bottom of the potential around the minimum point by a $2 \mathrm{D}$ harmonic potential, we find that the transverse oscillation frequencies are $\omega_{r} / 2 \pi \cong 533$ $\mathrm{KHz}$ and $\omega_{\varphi} / 2 \pi \cong 156 \mathrm{KHz}$. These frequencies provide a ground-state localization of $l_{r} \cong 8.4 \mathrm{~nm}$ and $l_{\varphi} \cong 15.6$ nm.

\section{CONCLUSIONS}

We have demonstrated that a subwavelength-diameter optical fiber carrying a red-detuned light and a bluedetuned light can be used to trap and guide atoms outside the fiber. We have shown that, when both the input light fields are of circular polarization, a set of trapping minima of the potential in the transverse plane is formed as a ring around the fiber. In this case, the atoms can be confined to a cylindrical shell around the fiber. When one or both of the input light fields are of linear polarization, the potential achieves its minimum value at two single points in the transverse plane. In this case, the atoms can be confined along two straight lines parallel to the fiber axis. Due to the thin thickness of the fiber, we can use far-off-resonance fields with substantially differing evanescent decay lengths to produce a net potential with a large depth, a large coherence time, and a large trap lifetime. For example, a $0.2-\mu$ m-radius silica fiber carrying $30 \mathrm{~mW}$ of $1.06-\mu \mathrm{m}$-wavelength light and $29 \mathrm{~mW}$ of $700-\mathrm{nm}$-wavelength light, both fields are circularly polarized at the input, gives for cesium atoms a trap depth of $2.9 \mathrm{mK}$, a coherence time of $32 \mathrm{~ms}$, and a recoil-heating-limited trap lifetime of $541 \mathrm{~s}$.

\section{Acknowledgments}

This work was carried out under the 21st Century COE program on "Coherent Optical Science".
[*] Also at Institute of Physics and Electronics, Vietnamese Academy of Science and Technology, Hanoi, Vietnam.

[1] S. Chu, Rev. Mod. Phys. 70, 685 (1998); C. CohenTannoudji, ibid. 70, 707 (1998); W.D. Phillips, ibid. 70, 721 (1998).

[2] C.S. Adams, M. Sigel, and J. Mlynek, Phys. Rep. 240, 143 (1994); V.I. Balykin and V.S. Letokhov, Atom Optics with Laser Light, Laser Science and Technology 18 (Harwood Academic, New York, 1995) p. 115; P. Meystre, Atom Optics (Springer, New York, 2001) p. 311.

[3] A.P. Kazantsev, G.J. Surdutovich, and V.P. Yakovlev, Mechanical Action of Light on Atoms (World Scientific, Singapore, 1990); R. Grimm, M. Weidemüller, and Yu.B. Ovchinnikov, Adv. At., Mol., Opt. Phys. 42, 95 (2000); V.I. Balykin, V.G. Minogin, and V.S. Letokhov, Rep. Prog. Phys. 63, 1429 (2000).

[4] R.J. Cook and R.K. Hill, Opt. Commun. 43, 258 (1982); V.I. Balykin, V.S. Letokhov, Yu.B. Ovchinnikov, and A. I. Sidorov, Phys. Rev. Lett. 60, 2137 (1988).

[5] For review see, for example, J.P. Dowling and J. GeaBanacloche, Adv. At., Mol., Opt. Phys. 37, 1 (1996); V.I. Balykin, ibid. 41, 181 (1999).

[6] M.A. Ol'Shanii, Yu.B. Ovchinnikov, and V.S. Letokhov, Opt. Commun. 98, 77 (1993).

[7] M.J. Renn, D. Montgomery, O. Vdovin, D.Z. Anderson, C.E. Wieman, and E.A. Cornell, Phys. Rev. Lett. 75, 3253 (1995); H. Ito, T. Nakata, K. Sakaki, M. Ohtsu, K.I. Lee, and W. Jhe, ibid. 76, 4500 (1996).

[8] V.I. Balykin, Fam Le Kien, J.Q. Liang, M. Morinaga, K. Hakuta, Atom spinning around an optical fiber: bound states and guiding, CLEO/IQEC and PhAST Technical Digest on CD-ROM (Optical Society of America, Washington, DC 2004), presentation ITuA7; V.I. Balykin, K. Hakuta, Fam Le Kien, J.Q. Liang, M. Morinaga, LANL e-print physics/0404110 (accepted for Phys. Rev. A).

[9] Yu.B. Ovchinnikov, S.V. Shul'ga, and V.I. Balykin, J. Phys. B 24, 3173 (1991).
[10] H. Mabuchi and H.J. Kimble, Opt. Lett. 19, 749 (1994).

[11] A.H. Barnett, S.P. Smith, M. Olshanni, K.S. Johnson, A.W. Adams, and M. Prentiss, Phys. Rev. A 61, 023608 (2000).

[12] J.P. Burke, Jr., Sai-Tak Chu, G.W. Bryant, C.J. Williams, and P.S. Julienne, Phys. Rev. A 65, 043411 (2002).

[13] L. Tong, R.R. Gattass, J.B. Ashcom, S. He, J. Lou, M. Shen, I. Maxwell, E. Mazur, Nature 426 (2003) 816.

[14] T.A. Birks, W.J. Wadsworth, P.St.J. Russell, Opt. Lett. 25 (2000) 1415; S.G. Leon-Saval, T.A. Birks, W.J. Wadsworth, P.St.J. Russell, M.W. Mason, Conference on Lasers and Electro-Optics (CLEO), Technical Digest, Postconference Edition (Optical Society of America, Washington, DC 2004), paper CPDA6.

[15] J. Bures, R. Ghosh, J. Opt. Soc. A 16 (1999) 1992.

[16] M.J. Levene, J. Korlach, S.W. Turner, M. Foquet, H.G. Craighead, and W.W. Webb, Science 299, 682 (2003).

[17] L. Tong, J. Lou, and E. Mazur, Optics Express 12, 1025 (2004).

[18] Fam Le Kien, J.Q. Liang, K. Hakuta, and V.I. Balykin (submitted to Opt. Commun.).

[19] J.C. Knight, G. Cheung, F. Jacques, and T.A. Birks, Opt. Lett. 22, 1129 (1997); M. Cai and K. Vahala, ibid. 26, 884 (2001).

[20] See, for example, A. Yariv, Optical Electronics (CBS College, New York, 1985); D. Marcuse, Light Transmission Optics (Krieger, Malabar, FL, 1989).

[21] See, for example, J.D. Jackson, Classical Electrodynamics, 3rd ed. (Wiley, New York, 1999).

[22] M. Boustimi, J. Baudon, P. Candori, and J. Robert, Phys. Rev. B 65, 155402 (2002); M. Boustimi, J. Baudon, and J. Robert, ibid. 67, 045407 (2003).

[23] G. Ghosh, Handbook of Thermo-Optic Coefficients of Optical Materials with Applications (Academic Press, New York, 1997).

[24] A.D. McLachlan, Mol. Phys. 7, 381 (1964). 
[25] J.E. Sansonetti, W.C. Martin, and S.L. Young, Handbook of Basic Atomic Spectroscopic Data (version 1.00). Online Available: http://physics.nist.gov/Handbook Na- tional Institute of Standards and Technology, Gaithersburg, MD. 